\title{
Short term clinical outcomes of accelerated hypofractionated radiotherapy in inoperable non-small cell lung cancer patients
}

\author{
Shoon Mya Aye ${ }^{\mathbb{O}}$, Lin Lin Kyi, Moe Hlaing, Aye Aye Myint, Khin Cho Win \\ Radiotherapy Department, Yangon General Hospital, Yangon, Myanmar
}

\begin{abstract}
Background: This study aimed to evaluate short term clinical outcomes of accelerated hypofractionated radiotherapy (AHRT) regarding locoregional response (LRR), symptoms relief and acute toxicities in non-small cell lung cancer (NSCLC) patients. The radical treatment for inoperable NSCLC is intolerable for some patients. An alternative RT regime should be considered for them.

Materials and methods: Inoperable NSCLC patients who could not tolerate radical treatment were treated with AHRT ( 45 Gy in 15 fractions over three weeks) by using the 3-dimensional conformal (RT) technique. The LRR was assessed by chest computed tomography (CT) performed before and 6 weeks after RT. Relief of symptoms such as cough, dyspnoea and chest pain was evaluated during RT and 6 and 12 weeks after RT, compared with the status before RT. Treatment-related acute toxicities such as dysphagia and radiation dermatitis were observed during and 6 and 12 weeks after RT.

Results: Total 65 patients (seven patients of stage II and fifty-eight patients of stage III) were included. Partial response was seen in $70.8 \%$ of patients, and stable disease was seen in $29.2 \%$ while there was neither complete response nor progressive disease after RT. Statistically significant associations were found between tumour response vs. pre-treatment tumour size and tumour response vs. performance status of the patients. Satisfactory symptom relief was found after RT, but severe acute dysphagia and radiation dermatitis (more than grade 3) were not observed.

Conclusion: Satisfactory LRR, symptom relief and acute toxicities were achieved by this regime. Long term studies are recommended to evaluate late toxicities and survival outcome further.

Trial registration no.: TCTR20200110001
\end{abstract}

Key words: accelerated hypofractionated radiotherapy; inoperable NSCLC; 3D-CRT; clinical outcome Rep Pract Oncol Radiother 2021;26(5):747-755

\section{Introduction}

According to the Global Cancer Observatory, the global cancer burden has increased to 18.1 million cases and 9.6 million cancer-related deaths in 2018. About $70 \%$ of cancer-related deaths occurred in low- and middle-income countries.
Among them, lung cancer is the most common type of cancer, representing $11.6 \%$ of all new cases and $18.4 \%$ of all cancer deaths [1]. In Radiotherapy department of the Yangon General Hospital, lung cancer was the second leading cause of morbidity in 2018. There were 1495 new lung cancer cases which accounted for about $19.9 \%$ of total patients.

Address for correspondence: Shoon Mya Aye, Radiotherapy Department, Yangon General Hospital, Yangon, Myanmar, No. 74, 7th floor, 13th Street (Middle), Lanmadaw Township, Yangon, Myanmar 11131, tel: +959977235193; e-mail: drshoonmyaaye89@gmail.com 
Compared to the previous data (1180 new lung cancer patients in 2017), the incidence has been rising annually. On the other hand, limitation of resources in a developing country like Myanmar has become a large barrier to overcome the massive burden of the disease.

The current standard treatment for medically or surgically inoperable non-small cell lung cancer (NSCLC) is once-daily radiation treatments of 60 Gy at 2 Gy per fraction, established by the Radiation Therapy Oncology Group (RTOG) 0617 trial [2]. The addition of concurrent chemotherapy can further improve the treatment outcome[3]. This standard concurrent chemoradiation (CCRT) given for six weeks may be difficult to tolerate for patients with comorbidities and poor performance status (PS). Therefore, those patients who are unfit for standard CCRT treatment should be treated with a shorter course radiation regime such as accelerated or hypofractionated regime. Accelerated radiotherapy schedules have the advantage of shortened overall treatment time to save time and costs as well as the benefit of counteracting rapid proliferation of tumour cells [4]. Hypofractionated radiotherapy consists of significantly fewer fractions of radiation, higher doses per fraction than conventional $2 \mathrm{~Gy}$, and lower cumulative doses. The use of larger than standard fraction causes a substantial risk of late toxicities in slowly proliferating tissues (spinal cord, lung, heart) that may be life-threatening [4]. However, the technological advances in radiotherapy treatment planning and delivery favour a wide use of large radiation fractions for both cure and palliation. By using hypofractionation, high doses per fraction are likely to achieve more cell kill per single exposure. Therefore, when the need for rapid symptom control and tumour shrinkage is greater than the risk of late toxic effects, a large fraction therapy may be preferred [4].

Radiotherapy dose fractionation schedule (2019) by the Royal College of Radiologists recommended high dose hypofractionated palliative regimes for patients with good PS because they confer moderate survival advantage at the expense of extra toxicity [5]. A Cochrane review also concluded that different palliative radiotherapy regimes equally palliate thoracic symptoms and patients with good PS should be considered for treatment with higher dose palliative regimes if there is a chance for a modest increase in survival [6]. According to
Amini et al. (2012) and Nguyen et al. (1999), accelerated hypofractionated radiation therapy (AHRT) with 45 Gy in 15 fractions over three weeks appears to be an acceptable treatment option which has similar efficacy without increasing toxicity when compared to conventional radiation $60 \mathrm{~Gy}$ in $2 \mathrm{~Gy}$ per fraction $[7,8]$.

Previously, inoperable non-small cell lung cancer patients of our department were treated with radiotherapy regime of $60 \mathrm{~Gy}$ in 30 fractions as curative schedule or 20 Gy in 5 fractions as palliative schedule, depending on the stage of lung cancer, PS and comorbid diseases of those patients. However, old patients with some comorbidities may have difficulties to complete the full course of $60 \mathrm{~Gy}$ in 30 fractions for six weeks. On the other side, patients with good PS should be treated with moderate hypofractionated schedule rather than $20 \mathrm{~Gy}$ in 5 fractions [9]. That is why this study was planned to assess the short term clinical outcomes such as LRR, symptom relief and acute toxicities of AHRT regime (45 Gy in 15 fractions over three weeks) by using $3 \mathrm{D}$ conformal planning given to inoperable NSCLC with ECOG PS $\leq 2$. Such an approach may be a reasonable and convenient option for inoperable NSCLC patients who could not achieve radical cure in a resource-limited country like Myanmar.

\section{Materials and methods}

This was a hospital-based prospective study done at the Radiotherapy Department, Yangon General Hospital (YGH), Myanmar. All cases of histologically proven and unresectable or medically inoperable NSCLC (stage I to III) patients who are unfit for chemotherapy due to comorbidities (e.g., poor cardiac, liver or renal function, etc. or old age) and patients with Eastern Cooperative Oncology Group (ECOG) PS 0, 1 or 2 were recruited between January 2018 and December 2018. Patients with distant metastasis and patients previously treated with thoracic radiotherapy or chemotherapy were excluded.

After selection according to the inclusion and exclusion criteria, written informed consent was taken before the study. Staging assessment was done with chest X-Ray (CXR), chest CT and ultrasonography (Abdomen \& pelvis). Bone scan and CT head or abdomen were done if symptoms 
for suspicious metastasis appeared. Staging of tumour followed the American Joint Committee on Cancer staging criteria (eighth edition) [10]. Any infection, such as chest infection, oral candidiasis or pulmonary $\mathrm{TB}$, was controlled first before radiotherapy.

\section{Radiotherapy technique}

Immobilization was done in a supine position with arms immobilized above the head in a comfortable, reproducible position to allow a greater choice of beam angle. CT scanning was obtained from the cricoid cartilage to the superior limit of the L2 vertebra with $3-5 \mathrm{~mm}$ slice interval. The parenchymal extent of the Gross tumour volume (GTV) was defined with CT images. The spiculated edge of the tumour and any involved node were included within the GTV. Clinical target volume (CTV) was an $8 \mathrm{~mm}$ margin from GTV. Planning target volume (PTV) margins were $10 \mathrm{~mm}$ from CTV for tumour motion and setup errors. An isocenter was tattooed in the CT scanner, as are lateral reference points. Elective nodal irradiation was not performed [11].

Three-dimensional conformal planning technique with the planning software "Eclipse" External Beam Planning 10.0.42 was used. To reduce lung dose, anterior oblique, posterior oblique and lateral beams were used. Wedges compensated for the obliquity of the beams in relation to the chest wall, and multileaf collimator shielding was used to conform each beam shape to the PTV. The V20 (volume of the lung minus PTV receiving more than 20Gy) was kept below 32 per cent to avoid pneumonitis. The length of oesophagus in the treated volume was kept below $8 \mathrm{~cm}$ by choosing beam angles carefully to prevent esophagitis [11]. Radiotherapy was administered with total $45 \mathrm{~Gy}$ at $3 \mathrm{~Gy}$ per fraction, 5 fractions per week, over 3 weeks, by a Linear Accelerator Machine (Clinac iX, Varian Medical Systems, USA).

All patients underwent chest CT at pre-treatment and six weeks after radiotherapy for assessing LRR (both tumour and lymph node changes). Revised RECIST (Response Evaluation Criteria in Solid Tumours) guideline version 1.1 was used to detect LRR [12]. Relief of symptoms and treatment-related acute toxicities (developed within 90 days after start of treatment) were assessed weekly during RT, six and twelve weeks after RT, compared to pretreatment baseline status. The assessment included cough, dyspnoea, chest pain, skin reaction and dysphagia. Common Terminology Criteria for Adverse Events (CTCAE) version 5.0 were used to study these symptoms and toxicities [13].

\section{Statistical analysis}

Continuous variables were described as mean \pm standard deviation (SD) and compared with the independent samples $t$ test. Categorical variables were described as a number and percentage and compared with the Chi-square or Fisher's exact test. Data analysis was performed on SPSS version 16 (SPSS Inc., 444 N. Michigan, Chicago, Illinois, USA).

\section{Results}

Among the recruited 65 patients, two patients were lost to follow-up at 12 weeks after RT due to non-cancer-related death. The baseline patients' characteristics were described in Table 1.

Table 1. Baseline patients' characteristics $(n=65)$

\begin{tabular}{|l|c|}
\hline \multicolumn{1}{|c|}{} & Mean \pm SD (range) \\
\hline Age (year) & $69.82 \pm 9.52(49-88)$ \\
\hline Sex & \\
Male & $45(69.23 \%)$ \\
Female & $20(30.77 \%)$ \\
\hline ECOG performance status & $10(15.38 \%)$ \\
0 & $29(44.62 \%)$ \\
1 & $26(40.00 \%)$ \\
\hline 2 & $14(67.69 \%)$ \\
\hline History of smoking & \\
\hline Histological types & $46(70.77 \%)$ \\
Squamous cell carcinoma & $5(7.69 \%)$ \\
Adenocarcinoma & $12(18.46 \%)$ \\
Large cell carcinoma & $2(3.08 \%)$ \\
Others & \\
\hline Staging & $7(10.77 \%)$ \\
IIB & $22(33.85 \%)$ \\
IIIA & $28(43.08 \%)$ \\
IIIB & $8(12.31 \%)$ \\
IIIC & $6.26 \pm 1.84(2.7-10)$ \\
\hline Tumour size [cm] & $1(1.54 \%)$ \\
$\leq 3 \mathrm{~cm}$ & $20(30.76 \%)$ \\
$3-5 \mathrm{~cm}$ & $24(36.92 \%)$ \\
$5-7$ cm & $20(30.76 \%)$ \\
$>7$ cm & \\
\hline
\end{tabular}




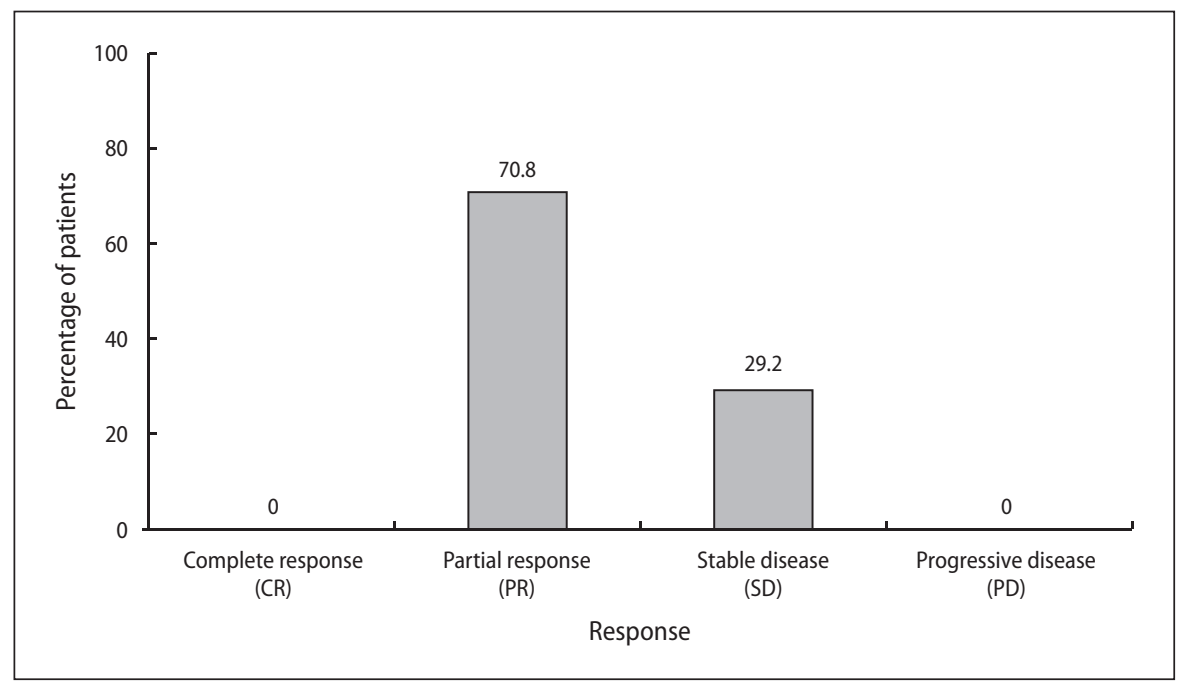

Figure 1. Locoregional response (LRR) of patients six weeks after RT $(n=65)$

\section{Locoregional response (LRR)}

The LRR of the patients six weeks after RT is presented in Figure 1. Partial response (PR) was seen in 46/65 (70.8\%) of patients and stable disease (SD) was seen in 19/65 (29.2\%) while there was neither a complete response (CR) nor a progressive disease (PD). One patient with SD was found to develop brain metastasis at 10 weeks after RT.

\section{Symptoms relief}

Grading of symptoms (cough, dyspnea and chest pain) at pre-RT, during RT and after RT was shown in Figure 2 (A, B, C). Patients who suffered from grade $2-3$ cough represented $63.08 \%$ of the total before RT, 3.08\% at 6 weeks after RT and $0 \%$ at 12 weeks after RT (Fig. 2A). It was found that patients who suffered from grade 2-3 dyspnoea accounted for $41.54 \%$ of the total before RT, $1.54 \%$ at 6 weeks after RT and $0 \%$ at 12 weeks after RT (Fig. 2B). The incidence of Grade 2-3 chest pain decreased from $59.99 \%$ before RT to $0 \%$ at the $3^{\text {rd }}$ week during RT and afterwards (Fig. 2C).

\section{Acute toxicities}

Table 2 showed the grading of dysphagia and radiation dermatitis during $\mathrm{RT}$ and after RT. Dysphagia was mostly found in the $3^{\text {rd }}$ week during RT (12.3\% for Grade 1 dysphagia and $4.62 \%$ for grade 2 dysphagia. This value decreased after RT gradually, and most patients (92.3\%) had no dysphagia at 12 weeks after RT. No severe dysphagia (more than grade 2) occurred. The majority of patients
(95.38\%) were free from radiation dermatitis. There were only 3 patients (4.62\%) who suffered from mild radiation dermatitis (grade 1). No severe radiation dermatitis (grade 2 and above) was found in the study.

\section{Associations between tumour responses and baseline patients' characteristics}

Association between tumour response and age, sex, stages and size of tumour and PS of the patients was described in Table 3. There was no statistically significant difference in age, sex and stages of tumour between partial responders and SD ( $p>0.05)$. The mean tumour size of partial responders was $5.65 \pm 1.62 \mathrm{~cm}$ and that of SD was $7.73 \pm 1.50$ $\mathrm{cm}$ and that difference was statistically significant with $\mathrm{p}<0.001$. The PS of patients before RT was also found to be statistically different between partial responders and SD $(\mathrm{p}<0.05)$. Among patients with PS $0-1$, the number of partial responders was higher than that of SD, whereas patients with SD were mostly found in the PS 2 group.

\section{Discussion}

In this study, the overall response rate (combined CR and PR) of $70.8 \%$ was achieved with the simple 3D conformal RT technique. Grade 2 dysphagia was mostly seen in the 3 rd week during RT, representing $4.62 \%$ of total patients. No severe esophageal toxicity ( $>$ grade 3 ) was seen, and no patient had $\geq$ grade 2 radiation dermatitis. A study done by 


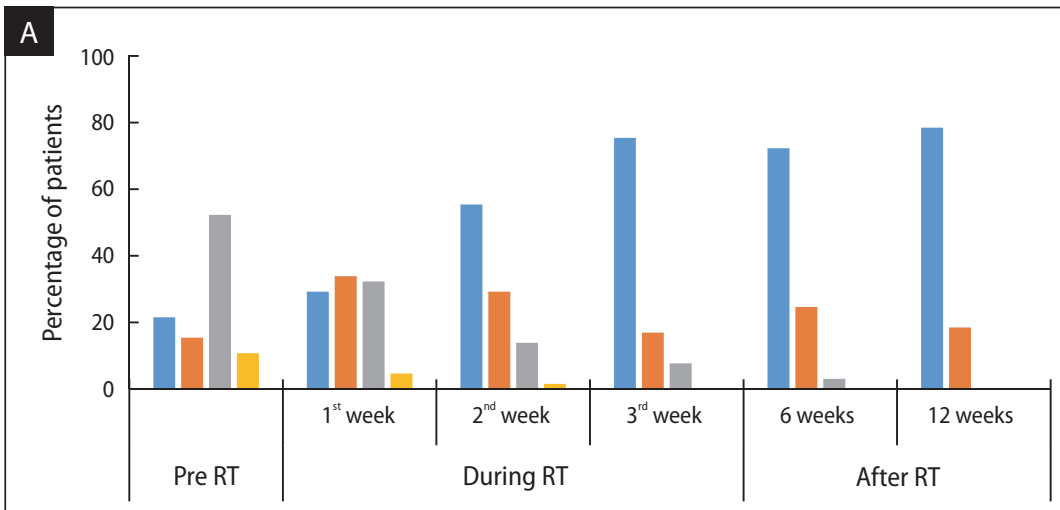

B

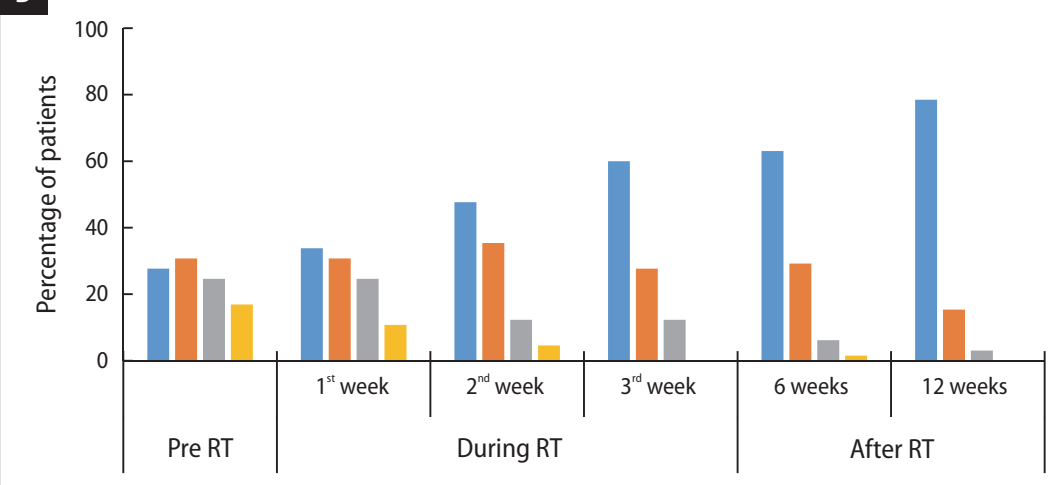

C

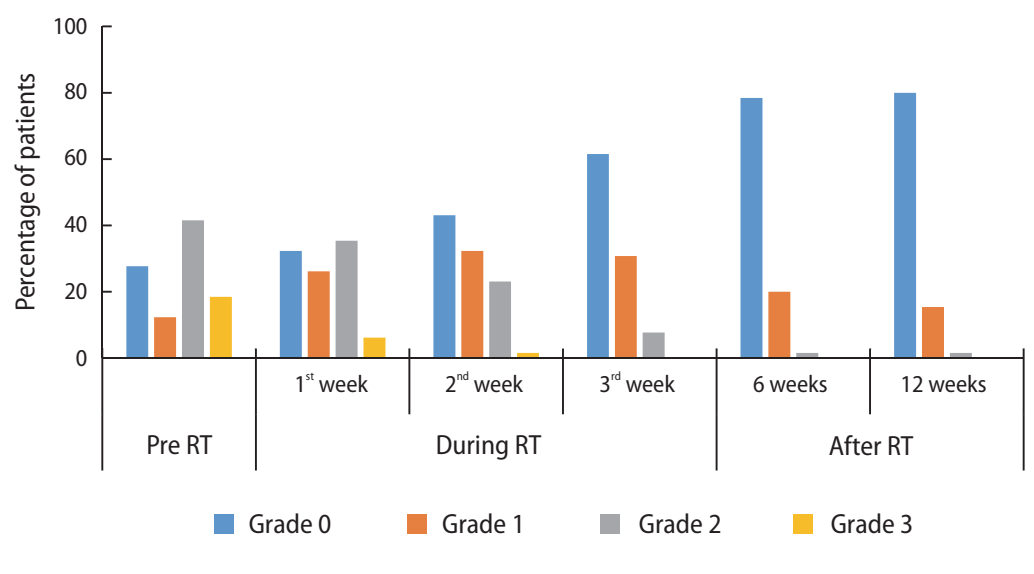

Figure 2. Grading of symptoms at pre-RT, during RT and after RT $(n=65)$. A. Cough; B. Dyspnea; C. Chest pain (2 missing values in 12 weeks after RT). RT — radiotherapy

Table 2. Grading of acute toxicities during radiotherapy (RT) and after RT

\begin{tabular}{|c|c|c|c|c|c|c|}
\hline \multirow{2}{*}{$\begin{array}{l}\text { Acute } \\
\text { toxicities }\end{array}$} & \multirow[t]{2}{*}{ Grades } & \multicolumn{3}{|c|}{$\begin{array}{c}\text { During RT } \\
\text { №. (\%) }\end{array}$} & \multirow{2}{*}{$\begin{array}{c}6 \text { weeks after RT } \\
\text { No. (\%) }\end{array}$} & \multirow{2}{*}{$\begin{array}{c}12 \text { weeks after } \text { RT* }^{*} \\
\text { No. }(\%)\end{array}$} \\
\hline & & $1^{\text {st }}$ week & $2^{\text {nd }}$ week & $3^{\text {rd }}$ week & & \\
\hline \multirow{3}{*}{ Dysphagia } & Grade 0 & 63 (96.92) & $60(92.31)$ & $54(83.08)$ & $58(89.23)$ & $60(92.31)$ \\
\hline & Grade 1 & $2(3.08)$ & $4(6.15)$ & $8(12.30)$ & $5(7.69)$ & $3(4.62)$ \\
\hline & Grade 2 & $0(0.00)$ & $1(1.54)$ & $3(4.62)$ & $2(3.1)$ & $0(0.00)$ \\
\hline \multirow{2}{*}{$\begin{array}{l}\text { Radiation } \\
\text { Dermatitis }\end{array}$} & Grade 0 & $65(100.00)$ & $65(100.00)$ & 62 (95.38) & 64 (98.46) & 63 (96.92) \\
\hline & Grade 1 & $0(0.00)$ & $0(0.00)$ & $3(4.62)$ & $1(1.54)$ & $0(0.00)$ \\
\hline
\end{tabular}

*2 missing values in 12 weeks after RT 
Table 3. Association between tumour response and baseline patients' characteristics

\begin{tabular}{|c|c|c|c|}
\hline & $\begin{array}{c}\text { Partial response } \\
\text { Mean } \pm \text { SD/No. }(\%) \\
(n=46)\end{array}$ & $\begin{array}{c}\text { Stable disease } \\
\text { Mean } \pm \text { SD/No. }(\%) \\
(n=19)\end{array}$ & p \\
\hline Age [years] & $70.46 \pm 10.21$ & $68.26 \pm 7.60$ & $0.403^{\#}$ \\
\hline $\begin{array}{l}\text { Sex } \\
\text { Male } \\
\text { Female }\end{array}$ & $\begin{array}{l}31(67.4) \\
15(32.6)\end{array}$ & $\begin{array}{c}14(73.7) \\
5(26.3)\end{array}$ & $0.617^{\wedge}$ \\
\hline $\begin{array}{l}\text { Stages } \\
\text { IIB } \\
\text { IIIA } \\
\text { IIIB } \\
\text { IIIC } \\
\end{array}$ & $\begin{array}{c}6(13) \\
18(39.1) \\
17(37) \\
5(10.9)\end{array}$ & $\begin{array}{c}1(5.3) \\
4(21.1) \\
11(57.9) \\
3(15.8)\end{array}$ & $0.329^{\$}$ \\
\hline Size of tumour [cm] & $5.65 \pm 1.62$ & $7.73 \pm 1.50$ & $0.00001^{\# * *}$ \\
\hline $\begin{array}{l}\text { Performance status } \\
0 \\
1 \\
2\end{array}$ & $\begin{array}{l}8(17.4) \\
25(54.3) \\
13(28.3)\end{array}$ & $\begin{array}{l}2(10.5) \\
4(21.1) \\
13(68.4)\end{array}$ & $0.010^{\wedge *}$ \\
\hline
\end{tabular}

${ }^{*} \mathrm{p}<0.05 ;{ }^{* *} \mathrm{p}<0.001 ;{ }^{*}$ independent samples $\mathrm{t}$ test; ${ }^{\wedge}$ Chi-square test; ${ }^{\mathrm{S}}$ Fisher's exact test

Nguyen et al. at the M.D. Anderson Cancer Center, Texas, reported that the response rate of AHRT regime with 45 Gy in 15 fractions in locally advanced inoperable NSCLC patients as 52\% (CR 14\% + PR $38 \%)$. Toxicity results were not described in that study [8]. A retrospective analysis of 300 patients with stage III NSCLC done at the same centre by Amini et al. reported the toxicity rates of the $45 \mathrm{~Gy}$ in 15 fractions regime were 107 (89.9\%) for grade 0 - 1 esophagitis, 12 (10.1\%) for grade $\geq 2$ esophagitis, 110(92.4\%) for Grade 0-1 dysphagia, 9 (7.6\%) for grade $\geq 2$ dysphagia and $2(1.7 \%)$ for grade $\geq 2$ radiation dermatitis [7]. A prospective randomized trial done in South Africa compared two radiotherapy regimes: 35 Gy in 10 fractions and 45 Gy in 15 fractions. The response rates were $56 \%$ (CR $12 \%+$ PR $44 \%)$ and 51\% (CR 10\% + PR 41\%), respectively. The incidence of moderate to severe radiation esophagitis was $23 \%$ and $41 \%$, respectively. 84 patients were included, and the 2D RT technique was used [14]. In a study by Teo et al., 45 Gy in 18 fractions was given to 128 NSCLC patients. The response rate was 53\% (no CR and 53\% PR), and radiation-induced esophagitis was found in $9 \%$ of patients. Skin reaction was not described [15].

The response rate of our study was superior to those achieved by Nguyen et al. (52\%) Abaratt et al. (51\%) and Teo et al. (53\%). The esophageal and skin toxicity rates were similar to those studies and not severe at all. Most of the patients in our study suffered from cough (78\%), dyspnoea (62\%) and chest pain (72\%) before RT. These symptoms were reduced after RT. Patients who suffered from grade $2-3$ cough were $63.08 \%$ of the total before RT, $3.08 \%$ at six weeks after RT and $0 \%$ at twelve weeks after RT. Similarly, the incidence of Grade 2-3 dyspnoea decreased from $41.54 \%$ before RT to $1.54 \%$ at six weeks after RT, and $0 \%$ at twelve weeks after RT. Grade 2-3 chest pain was experienced by $59.99 \%$ of patients before RT, and by none after RT. These results suggest that this regime could give an excellent symptomatic response in addition to a good radiologic response.

A phase 2 trial (ICORG 99-09) of dose-escalating AHRT in stage I-III NSCLC patients showed that 72 Gy in 24 fractions (3Gy per fraction) gave the response rate of $34 \%(20 \% \mathrm{CR}+14 \% \mathrm{PR})$, $\geq$ grade 2 acute esophageal toxicity of $27 \%, \geq$ grade 2 acute lung toxicity of $9 \%, \geq$ grade 2 late esophageal toxicity of $14 \%, \geq$ grade 2 late lung toxicity of $23 \%$, and $\geq$ grade 2 acute skin reaction of $23 \%$ [16]. Another retrospective study of the AHRT regime with 55 Gy in 20 fractions in four UK centers resulted in CR (24.3\%), PR (46.3\%), SD (13.8\%), PD $(2.6 \%)$ and unknown (13.0\%). The median overall survival was 24 months from time of diagnosis and 2 -year overall survival was $50 \%$. No grade 3-4 toxicities were observed [17]. A long-term follow-up analysis done in Italy reported that image-guided hypofractionated radiotherapy (60Gy /20 fractions 
with 3 Gy per fractions) given to sixty patients with advanced-stage NSCLC (IIIA-IV) achieved CR $10 \%$ and PR $76 \%$. Among them, $27 \%$ of patients had $>$ grade 2 esophagitis. Radiation-induced skin reaction was found in $8 \%$ of patients and all were Grade 1 [18]. In comparison with those AHRT regimes with higher doses, the regime of our study resulted in similar LRR and lower toxicity outcomes. Incorporating other new technologies, such as PET/CT image fusion for radiation treatment planning, respiratory gating or respiratory correlated radiation treatment, intensity-modulated radiotherapy and image-guided radiotherapy may further reduce the likelihood of this hypofractionated regimen inducing toxicities. It would also possibly allow further dose escalation.

In Myanmar, there is still a paucity of data about hypofractionated radiotherapy in lung cancer cases. In a clinical study done in YGH, short course palliative hypofractionated radiotherapy with $20 \mathrm{~Gy}$ in 5 fractions was given to 48 patients of stage IIIB-IV NSCLC. It resulted in a 56\% response rate (no CR+ $56 \%$ PR), 20\% PD and 24\% SD. Patients suffered from Grade 1 dysphagia in 20\% of cases and Grade 2 dysphagia in $2 \%$ of cases [19]. In another study of inoperable NSCLC patients done in YGH, the LRR rate of sequential chemoradiation with palliative RT (30 Gy in 10 fractions) given to 28 cases of stage IIIB was $12 \%$ (no CR $+12 \%$ PR). $15 \%$ of patients had SD, and 1\% had PD [20]. Compared to those studies, the AHRT regime of 45 Gy in 15 fractions over three weeks gave a better response rate and similar toxicity outcomes.

While concurrent chemoradiotherapy (CCRT) has been known to be the standard treatment of NSCLC patients, patients with old age, poor PS or some comorbidities rarely completed the full course CCRT regime within long duration, leading to treatment interruptions and subsequently reducing the survival outcomes. To overcome these difficulties, the AHRT regime could be a solution for patients who are unfit for standard CCRT. In our study, 45 Gy in 15 fractions given to inoperable NSCLC patients achieved an acceptable response rate with acceptable acute toxicity. Although this study achieved a good overall response rate of $71 \%$, no CR was seen. This may be related to the fact that we only selected cases who were unfit for chemotherapy. There has been strong evidence that combined chemoradiotherapy produces better outcomes than radiation alone [21]. Nevertheless, a study done by Trovo et al. (1990) reported that there was no significant difference in response rate between combined chemoradiotherapy and radiotherapy alone with the regime of 45 Gy in 15 fractions $(38.8 \%$ vs. $56.4 \%)(\mathrm{p}=0.900)$ [22].

In our study, statistically significant relationships were found between the LRR of the tumour to radiotherapy with pre-treatment tumour size $(\mathrm{p}<0.001)$ and with PS of the patient $(\mathrm{p}<0.05)$. A Cochrane systematic review about radical radiotherapy for medically inoperable NSCLC patients concluded that better response rates and survival were seen in those with smaller tumour and those receiving higher radiation doses [23]. Other studies have already reported that tumour size has a significant impact on overall survival, leading to inferior survival if the primary tumour size is large [24]. Previous studies have shown that PS is associated with improved outcomes in NSCLC patients $[25,26]$.

There are several radiobiological benefits of the $45 \mathrm{~Gy}$ in 15 fraction treatment regime. Applying the $\alpha / \beta$ ratio of 10 , this regimen has a biological equivalent dose (BED10) of 58.5 Gy. Although this is lower than the BED10 for patients treated with conventionally fractionated radiation of 60-70 Gy (72-84 Gy), the data published by Nguyen et al. and Amini et al. indicate that control rates seem similar between the two treatment schedules (45 Gy in 15 fractions vs. 60 Gy in 30 fractions) [7, 8]. The benefit may come in the shortened treatment time of this AHRT regime. Lung cancer cells demonstrate accelerated repopulation during radiotherapy, and increases in treatment duration can, therefore, reduce local control, negatively impacting survival. Therefore, it is critical to reduce the overall treatment duration [27].

Another proved benefit of this RT regime is survival advantage and good tumour control which is comparable with conventional standard radiotherapy $[7,8]$. Radiotherapy dose fractionation schedule (2019) by the Royal College of Radiologists recommended high dose hypofractionated palliative regimes for patients with good PS because they confer moderate survival advantage at the expense of extra toxicity [5]. However, survival outcome could not be assessed in this study due to time limitation and feasibility reasons. Though the survival data could not be obtained within the limited 
study period, good LRR and symptom control had been achieved. From these outcomes, acceptable survival results could be expected, as tumour response was associated with better overall survival and progression-free survival in non-small cell lung cancer patients $[28,29]$.

There were two patients lost to follow up at twelve weeks after RT due to non-cancer-related death. A patient with brain metastasis was found at 10 weeks after RT and his LRR after RT was SD. As lung cancer has the nature of high rate of distant metastasis, the occurrence of brain metastasis may be due to distant failure or occult micrometastasis at the time of diagnosis. Apart from that, no other patient had symptoms indicating any local recurrence or distant failure.

\section{Conclusion}

This AHRT regime using 45 Gy in 15 fractions offers acceptable short term clinical outcomes such as good LRR, satisfactory symptom relief and well-tolerated acute toxicities for unresectable or medically inoperable NSCLC patients. Shorter treatment time than conventional fractionation can also prove more convenient and cost-effective. It might be a reasonable alternative for patients who are not candidates for conventional radiotherapy. Further long-term studies are warranted to find out the survival outcomes and late toxicities of this radiotherapy regime.

\section{Conflict of interest}

None declared.

\section{Funding}

None declared.

\section{Ethics approval}

This study was approved by the Postgraduate Board of Studies (Radiation Oncology), University of Medicine 1, Yangon, and it was registered at the Thai Clinical Trial Registry with the trial registration number TCTR20200110001. All procedures performed in this study were in accordance with the ethical standards of the institutional and/or national research committee and with the $1964 \mathrm{Hel}-$ sinki declaration and its later amendments or comparable ethical standards. Written informed consent was obtained from all individual participants included in the study. All authors have approved the final article.

\section{Acknowledgments}

We wish to thank Prof. Soe Oo Maung, Prof. Win Naing and consultants, seniors, colleagues, physicists and technicians from the Radiotherapy Department, Yangon General Hospital for their kind help and advice.

\section{References}

1. International Agency for Research On Cancer (IARC). GLOBOCAN 2018: Estimated Cancer Incidence, Mortality and Prevalence Worldwide in 2018. http://globocan.iarc. $\mathrm{fr} /$ old/FactSheets/cancers/lung-new.asp (20 July 2019).

2. Bradley JD, Paulus R, Komaki R, et al. Standard-dose versus high-dose conformal radiotherapy with concurrent and consolidation carboplatin plus paclitaxel with or without cetuximab for patients with stage IIIA or IIIB non-small-cell lung cancer (RTOG 0617): a randomised, two-by-two factorial phase 3 study. Lancet Oncol. 2015; 16(2): 187-199, doi: 10.1016/S1470-2045(14)71207-0, indexed in Pubmed: 25601342.

3. Curran WJ, Paulus R, Langer CJ, et al. Sequential vs. concurrent chemoradiation for stage III non-small cell lung cancer: randomized phase III trial RTOG 9410. J Natl Cancer Inst. 2011; 103(19): 1452-1460, doi: 10.1093/jnci/djr325, indexed in Pubmed: 21903745.

4. Steel G. Basic Clinical Radiobiology: Radiation response and tolerance of normal tissues. 3rd ed. Arnold, London 2002.

5. Royal College of Radiologists (RCR). Radiotherapy Dose Fractionation. 3rd ed. London 2019.

6. Lester JF, Macbeth FR, Toy E, et al. Palliative radiotherapy regimens for non-small cell lung cancer. Cochrane Database Syst Rev. 2006(4): CD002143, doi: 10.1002/14651858. CD002143.pub2, indexed in Pubmed: 17054152.

7. Amini A, Lin SH, Wei C, et al. Accelerated hypofractionated radiation therapy compared to conventionally fractionated radiation therapy for the treatment of inoperable non-small cell lung cancer. Radiat Oncol. 2012; 7: 33, doi: 10.1186/1748-717X-7-33, indexed in Pubmed: 22420631.

8. Nguyen L, Komaki R, Allen P, et al. Effectiveness of accelerated radiotherapy for patients with inoperable non-small cell lung cancer (NSCLC) and borderline prognostic factors without distant metastasis: a retrospective review. Int J Radiat Oncol Biol Phys. 1999; 44(5): 1053-1056, doi: 10.1016/ s0360-3016(99)00130-3, indexed in Pubmed: 10421538.

9. van Oorschot B, Assenbrunner B, Schuler M, et al. Survival and prognostic factors after moderately hypofractionated palliative thoracic radiotherapy for non-small cell lung cancer. Strahlenther Onkol. 2014; 190(3): 270-275, doi: 10.1007/s00066-013-0507-y, indexed in Pubmed: 24413894.

10. Rami-Porta R, Asamura H, Travis WD, Rusch VW. Lung. In: Rami-Porta R, Asamura H, Travis WD, Rusch VW. American Joint Committee on Cancer (AJCC). ed. AJCC Cancer Staging manual. 8th ed. Springer, New York 2017: 431-456. 
11. Barette A, Dobbs J, Morris S, Roques T. Practical radiotherapy planning. 4th ed. Hodder Arnold, Italy 2009.

12. Eisenhauer EA, Therasse P, Bogaerts J, et al. New response evaluation criteria in solid tumours: revised RECIST guideline (version 1.1). Eur J Cancer. 2009; 45(2): 228-247, doi: 10.1016/j.ejca.2008.10.026, indexed in Pubmed: 19097774.

13. National Cancer Institute (NCI). Common Terminology Criteria for Adverse Events (CTCAE) version 5.0. US Department of Health and Human Services, National Institute of hHealth, National Cancer Institute 2009.

14. Abratt RP, Shepherd LJ, Salton DG. Palliative radiation for stage 3 non-small cell lung cancer--a prospective study of two moderately high dose regimens. Lung Cancer. 1995; 13(2): 137-143, doi: 10.1016/0169-5002(95)00487-4, indexed in Pubmed: 8581393.

15. Teo P, Tai TH, Choy D, et al. A randomized study on palliative radiation therapy for inoperable non small cell carcinoma of the lung. Int J Radiat Oncol Biol Phys . 1988; 14(5): 867-871, doi: 10.1016/0360-3016(88)90007-7, indexed in Pubmed: 2452146.

16. Cagney DN, Thirion PG, Dunne MT, et al. A Phase II Toxicity End Point Trial (ICORG 99-09) of Accelerated Dose-escalated Hypofractionated Radiation in Nonsmall Cell Lung Cancer. Clin Oncol (R Coll Radiol). 2018; 30(1): 30-38, doi: 10.1016/j.clon.2017.10.010, indexed in Pubmed: 29097074.

17. Din OS, Harden SV, Hudson E, et al. Accelerated hypofractionated radiotherapy for non small cell lung cancer: results from 4 UK centres. Radiother Oncol. 2013; 109(1): 8-12, doi: 10.1016/j.radonc.2013.07.014, indexed in Pubmed: 24094626.

18. Osti MF, Agolli L, Valeriani M, et al. Image guided hypofractionated 3-dimensional radiation therapy in patients with inoperable advanced stage non-small cell lung cancer. Int J Radiat Oncol Biol Phys. 2013; 85(3): e157-e163, doi: 10.1016/j.ijrobp.2012.10.012, indexed in Pubmed: 23182393.

19. Thida-Htun Y. Palliative effect of hypofractionated radiotherapy for advanced lung cancer. M.Med.Sc. Radiation Oncology) dissertation, University of Medicine 1, Yangon 2017.

20. Thuzar-Pyone K. Locoregional response of sequential chemoradiation for inoperable non-small cell lung cancer. M.Med.Sc. (Radiation Oncology) dissertation. University of Medicine 1, Yangon 2016.

21. Chiang A, Detterbeck FC, Stewart T, Decker RH, Tanoue L. Non-small-cell Lung Cancer . In: Devita VTVT, LawrenceTS,
Rosenberg SA. ed. Cancer: Principles and Practice of Oncology. 11th ed. Wolters Kluwer, London 2019: 1135-1221.

22. Trovó $M$, Minatel $E$, Veronesi A, et al. Combined radiotherapy and chemotherapy versus radiotherapy alone in locally advanced epidermoid bronchogenic carcinoma a randomized study. Cancer. 1990; 65(3): 400-404, doi: 10.1002/1097-0142(19900201)65:3<400::aidcncr2820650304>3.0.co;2-h, indexed in Pubmed: 2404555.

23. Rowell NP, Williams CJ. Radical radiotherapy for stage I/II non-small cell lung cancer in patients not sufficiently fit for or declining surgery (medically inoperable): a systematic review. Thorax. 2001; 56(8): 628-638, doi: 10.1136/ thorax.56.8.628, indexed in Pubmed: 11462066.

24. Zhang J, Gold KA, Lin HY, et al. Relationship between tumor size and survival in non-small-cell lung cancer (NSCLC): an analysis of the surveillance, epidemiology, and end results (SEER) registry. JThorac Oncol. 2015; 10(4): 682-690, doi: 10.1097/JTO.0000000000000456, indexed in Pubmed: 25590605.

25. Albain KS, Crowley JJ, LeBlanc M, et al. Survival determinants in extensive-stage non-small-cell lung cancer: the Southwest Oncology Group experience. J Clin Oncol. 1991; 9(9): 1618-1626, doi: 10.1200/JCO.1991.9.9.1618, indexed in Pubmed: 1651993.

26. Espinosa E, Feliu J, Zamora P, et al. Serum albumin and other prognostic factors related to response and survival in patients with advanced non-small cell lung cancer. Lung Cancer. 1995; 12(1-2): 67-76, doi: 10.1016/01695002(95)00407-r, indexed in Pubmed: 7600032.

27. Koukourakis $M$, Hlouverakis $G$, Kosma L, et al. The impact of overall treatment time on the results of radiotherapy for nonsmall cell lung carcinoma. Int J Radiat Oncol Biol Phys. 1996; 34(2): 315-322, doi: 10.1016/0360-3016(95)02102-7, indexed in Pubmed: 8567332.

28. Valeriani M, Marinelli L, Reverberi C, et al. Inoperable early-stage primary and early recurrent non-small cell lung cancer: outcomes of a mono-institutional experience using a moderate hypofractionated schedule. Radiol Med. 2019; 124(1): 58-64, doi: 10.1007/s11547-018-0934-z, indexed in Pubmed: 30178173.

29. Ball D, Mitchell A, Giroux D, et al. IASLC Staging Committee and Participating Institutions. Effect of tumor size on prognosis in patients treated with radical radiotherapy or chemoradiotherapy for non-small cell lung cancer. An analysis of the staging project database of the International Association for the Study of Lung Cancer. JThorac Oncol. 2013;8(3): 315-321, doi: 10.1097/JTO.0b013e31827dc74d, indexed in Pubmed: 23334092. 